\title{
A Partial Entropic Lattice Boltzmann MHD Simulation of the Orszag-Tang Vortex
}

\author{
Christopher Flint and George Vahala \\ Department of Physics, College of William \& Mary, Williamsburg, Virginia 23185
}

October 2, 2018

\begin{abstract}
Karlin has introduced an analytically-determined entropic lattice Boltzmann algorithm for Navier-Stokes turbulence. Here this is partially extended to a lattice Boltzmann model of magnetohydrodynamics, on using the vector distribution function approach of Dellar for the magnetic field. (which is permitted to have field reversal). The partial entropic algorithm is benchmarked successfully against standard simulations of the Orszag-Tang vortex 1].
\end{abstract}

\section{Introduction}

Because of its inherent simplicity and ease of being extremely parallizeable, the lattice Boltzmann (LB) algorithm has had considerable impact as a computational tool in the solution of NavierStokes flows 2. Interestingly, even though it is strictly a second-order accurate algorithm, in many applications its accuracy is usually comparable to the pseudo-spectral method. The Achilles' heel of simple LB is that it is prone to numerical instabilities for high Reynolds turbulent flows. This is attributable to the lack of an inherent mechanism to require the time evolution of the LB distribution function to remain non-negative.

By generalizing the simple single-relaxation-time (SRT) LB collision operator to incorporate multi-relaxation rates (MRT) [3 [5], one gained some new degrees of freedom that could be exploited to attain greater numerical instability. However there was no systematic way to constrain these extra relaxation rates, but their tuning was not only problem dependent but could also influence the viscsoity of the flow and hence the actual Reynolds number. An alternate and systematic approach to stabilizing LB is through an entropic principle and its assocaited H-theorem. $6[-22]$. In its current state, the entropic approach can be viewed as an optimized MRT algorithm in which emphasis is placed on an algebraically determined entropy stabilizing parameter that is not directly dependent on the MRT collisional rates and which does not affect the fluid viscosity. Hence these entropic simulations will be run at the same Reynolds number as the normal CFD computation, rather than at an augmented transport coefficient.

In generalizing the Karlin entropic algorithm to LB-MHD one must decide on the importance of avoiding divergence cleaning (by using a vector magnetic distribution 23]) over a scalar magnetic distribution that would permit magnetic field reversal. Here we choose to continue with the vector magnetic distribution representation in which the zeroth moment will yield the magnetic 
field $\vec{B}$. We are thus excluded from applying an extend entropic principle to the evolution of the vector distribution function. However there is some residual stabilization effects on the LB-MHD algorithm since the magnetic field appears in the fluid momentum equation to which there is applied an entropic ansatz. It should also be noted that this entropic stabilization parameter will be dynamically determined analytically throughout the simulation whereas the MRT relaxation rates are static and are not changed throughout the simulation. Moreover the entropic algorithm will permit simulations at arbitrary small viscosities - a regime where the static MRT algorithms cannot attain.

A moment-based representation for LB-MHD is given in Section 2, while the partial entropic algorithm is presented in Sec. 3. Since 2D and 3D MHD exhibit the same cascading spectra (e.g., energy cascades to high wavenumbers), it is convenient to test our partial entropic LB-MHD algorithm for the 2D Orszag-Teng vortex. Our simulations are compared to those of Orszag-Tang in Sec. 4

\section{Moment Basis Representation for Multiple Relaxation Model of LB-MHD}

There are several MRT extensions [14, 18, 21,24 of the original SRT LB-MHD model of Dellar 23]. However, for simplicity, we shall restrict ourselves to an SRT model for the vector magnetic field distribution $\vec{g}_{i}$, and an MRT model for the scalar distribution function $f_{i}$

$$
\begin{gathered}
\left(\partial_{t}+\partial_{\gamma} c_{\gamma i}\right) f_{i}=\sum_{j} X_{i j}^{\prime}\left(f_{j}^{(\mathrm{eq})}-f_{j}\right) \\
\left(\partial_{t}+\partial_{\gamma} c_{\gamma i}\right) \vec{g}_{i}=Y^{\prime}\left(\vec{g}_{i}^{(\mathrm{eq})}-\vec{g}_{i}\right)
\end{gathered}
$$

We employ the summation convention over the Greek indices which represent the vector nature of the fields $(\gamma=1,2$ for $2 \mathrm{D})$, while the summation over the Roman indices $i,(i=0 \ldots 8)$ for a $2 \mathrm{D}$ lattice, will always be made explicit. The relevant moments are

$$
\sum_{i} f_{i}=\rho \quad, \quad \sum_{i} f_{i} \vec{c}_{i}=\rho \vec{u} \quad \text { and } \quad \sum_{i} \vec{g}_{i}=\vec{B}
$$

and the positive definiteness of the $\vec{g}_{i}$ is lost for magnetic field reversal problems and thus precludes an explicit attempt at an entropy principle for $\vec{g}_{i} . X_{i j}^{\prime}$ is the MRT collision operator for the evolution of $f_{i}$ while $Y^{\prime}$ is the SRT for the evolution of $\vec{g}_{i}$. The MHD viscosity and resistivity transport coefficients are determined from some of these kinetic relaxation rates.

Since the mean velocity $\vec{u}$ is defined by the 1 st moment of the $f_{i}$, while the magnetic field $\vec{B}$ is determined from the 0th moment of the $\vec{g}_{i}$, a minimal LB representation of MHD on a square lattice requires a 9-bit model for the $f_{i}$ but just a 5 -bit model for the $\vec{g}_{i}$. However we find it more convenient to use a 9-bit lattice for both distributions, Fig.1.

To recover the MHD equations in the Chapman-Enskog limit, an appropriate choice of relaxation distribution functions $f_{i}^{(e q)}$ and $\vec{g}_{i}^{(e q)}$ is (where $w_{i}$ are appropriate weights, given in Fig. 1)

$$
\begin{gathered}
f_{i}^{(\mathrm{eq})}=w_{i} \rho\left[1+3\left(\vec{c}_{i} \cdot \vec{u}\right)+\frac{9}{2}\left(\vec{c}_{i} \cdot \vec{u}\right)^{2}-\frac{3}{2} \vec{u}^{2}\right]+\frac{9}{2} w_{i}\left[\frac{1}{2} \vec{B}^{2} \vec{c}_{i}^{2}-\left(\vec{B} \cdot \vec{c}_{i}\right)^{2}\right], i=0, . ., 8 \\
\vec{g}_{i}^{(\mathrm{eq})}=w_{i}\left[\vec{B}+3\left\{\left(\vec{c}_{i} \cdot \vec{u}\right) \vec{B}-\left(\vec{c}_{i} \cdot \vec{B}\right) \vec{u}\right\}\right], i=0, . ., 8
\end{gathered}
$$




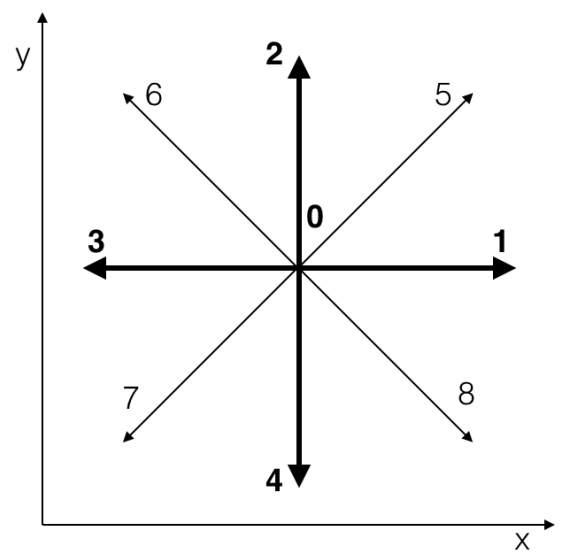

Figure 1: The kinetic lattice vectors for 2D LB-MHD in D2Q9: $\vec{c}_{i}=(0,0),(0, \pm 1),( \pm 1,0),( \pm 1, \pm 1)$. $w_{i}$ are appropriate weight factors dependent on the choice of lattice: for $D 2 Q 9, w_{0}=\frac{4}{9}$; for speed $1, w_{i}=\frac{1}{9}$; and for speed $\sqrt{2}, w_{i}=\frac{1}{36}$.

The LB-MHD equations are typically solved by an operator-splitting method: streaming and local collisional relaxation. The excellent parallelization follows from this: the streaming operation is a simple shift of the data from one lattice point to another, while the collision step is a purely local operation with its evaluation requires only data from only that grid point. MPI is only required when the data is streamed from one processor domain to another - and this is well parallelized since it is also synchronized throughout the lattice boundaries. The computationally difficult nonlinear convective derivatives $\vec{u} \cdot \nabla \vec{u}, \vec{u} \cdot \nabla \vec{B}, \vec{B} \cdot \nabla \vec{u}$ and $\vec{B} \cdot \nabla \vec{B}$ are replaced in LB by simple linear advection and local polynomial nonlinearities in $f_{i}^{(e q)}(\vec{u}, \vec{B})$ and $\vec{g}_{k}^{(e q)}(\vec{u}, \vec{B})$.

Since the collisional relaxation involves the local conservation of mass and momentum at each lattice site, it is convenient in MRT-LB to perform the collision step in moment space. The streaming is easiest to perform in distribution space $f_{i}, \vec{g}_{i}$. Thus for the moment basis one obviously chooses the conservation moments (the 0th and 1st moments of the $f_{i}$ and the 0th moment of $\left.\vec{g}_{i}\right)$. The choice of the remaining higher moments is somewhat arbitrary [25, 26]. There is a 1-1 (constant) transformation $\mathrm{T}$ between these spaces. We choose the same constant $9 \times 9 \mathrm{~T}$ - matrix to connect the scalar distributions $\left(f_{i}, i=0 . .8\right)$ to their moments $\left(M_{i}, i=0 . .8\right)$ as for the vector magnetic distributions $\left(\vec{g}_{i}, i=0 . .8\right)$ with their moments $\left(\vec{N}_{i}, i=0 . .8\right)$

$$
M_{i}=\sum_{j=0}^{8} \mathrm{~T}_{i j} f_{j} \quad, \quad \vec{N}_{i}=\sum_{q=0}^{8} \mathrm{~T}_{i q} \vec{g}_{q}
$$


where

$$
\mathrm{T}=\left(\begin{array}{c}
\mathbf{1} \\
c_{x} \\
c_{y} \\
c_{x} c_{y} \\
c_{x}^{2} \\
c_{y}^{2} \\
c_{x}^{2} c_{y} \\
c_{x} c_{y}^{2} \\
c_{x}^{2} c_{y}^{2}
\end{array}\right)=\left(\begin{array}{rrrrrrrrr}
1 & 1 & 1 & 1 & 1 & 1 & 1 & 1 & 1 \\
0 & 1 & 0 & -1 & 0 & 1 & -1 & -1 & 1 \\
0 & 0 & 1 & 0 & -1 & 1 & 1 & -1 & -1 \\
0 & 0 & 0 & 0 & 0 & 1 & -1 & 1 & -1 \\
0 & 1 & 0 & 1 & 0 & 1 & 1 & 1 & 1 \\
0 & 0 & 1 & 0 & 1 & 1 & 1 & 1 & 1 \\
0 & 0 & 0 & 0 & 0 & 1 & 1 & -1 & -1 \\
0 & 0 & 0 & 0 & 0 & 1 & -1 & -1 & 1 \\
0 & 0 & 0 & 0 & 0 & 1 & 1 & 1 & 1
\end{array}\right)
$$

The Cartesian components of the corresponding 9-dimensional lattice vectors are just

$$
c_{x}=\{0,1,0,-1,0,1,-1,-1,1\} \quad, \quad c_{y}=\{0,0,1,0,-1,1,1,-1,-1\} .
$$

For the scalar distributions, the $1^{\text {st }}$ row of the T-matrix is just the conservation of density while the $2^{\text {nd }}$ and $3^{\text {rd }}$ rows are just the conservation of momentum (2D). For the vector magnetic distributions the $1^{\text {st }}$ row of the T-matrix is the only collisional invariant.

With this moment basis, the MRT collisional relaxation rate tensor $X_{i j}^{\prime}$ is diagonalized with the $T$ - matrix as a similarity transformation. It is convenient to denote this diagonal matrix with elements $X_{i} \delta_{i j}$.

In the $D 2 Q 9$ phase space, the relaxation rate $X_{j}$ is associated with the corresponding moment $M_{j}, j=0 . .8$. Similarly for the magnetic distributions in SRT, there is just a single collisional

relaxation rate for each magnetic moment $\vec{N}_{k}$, and this will be denoted by $Y$. In particular, the equilibrium moments can be written in terms of the conserved moments:

$$
\begin{aligned}
& M_{0}^{(\mathrm{eq})}=M_{0}=\rho \quad M_{1}^{(\mathrm{eq})}=M_{1}=\rho u_{x} \quad M_{2}^{(\mathrm{eq})}=M_{2}=\rho u_{y} \\
& M_{3}^{(\mathrm{eq})}=\rho u_{x} u_{y}-B_{x} B_{y} \quad M_{4}^{(\mathrm{eq})}=\frac{1}{6}\left(6 \rho u_{x}^{2}+2 \rho-3\left(B_{x}^{2}-B_{y}^{2}\right)\right) \\
& M_{5}^{\text {(eq) }}=\frac{1}{6}\left(6 \rho u_{y}^{2}+2 \rho+3\left(B_{x}^{2}-B_{y}^{2}\right)\right) \quad M_{6}^{(\mathrm{eq})}=\frac{1}{3} \rho u_{y} \\
& M_{7}^{(\mathrm{eq})}=\frac{1}{3} \rho u_{x} \quad M_{8}^{(\mathrm{eq})}=\frac{1}{9} \rho\left(1+3 u_{x}^{2}+3 u_{y}^{2}\right) \\
& N_{\alpha 0}^{(\mathrm{eq})}=N_{\alpha 0}=B_{\alpha} \quad N_{\alpha 1}^{(\mathrm{eq})}=u_{x} B_{\alpha}-u_{\alpha} B_{x} \quad N_{\alpha 2}^{(\mathrm{eq})}=u_{y} B_{\alpha}-u_{\alpha} B_{y} \\
& N_{\alpha 3}^{\text {(eq) }}=0 \quad N_{\alpha 4}^{(\text {eq })}=\frac{B_{\alpha}}{3} \quad N_{\alpha 5}^{\text {(eq) }}=\frac{B_{\alpha}}{3} \\
& N_{\alpha 6}^{(\text {eq })}=\frac{1}{3}\left(u_{y} B_{\alpha}-u_{\alpha} B_{y}\right) \quad N_{\alpha 7}^{(\text {eq })}=\frac{1}{3}\left(u_{x} B_{\alpha}-u_{\alpha} B_{x}\right) \quad N_{\alpha 8}^{(\text {eq })}=\frac{B_{\alpha}}{9}, \quad \alpha=x, y
\end{aligned}
$$

\section{Entropic method [6-15] and its partial extension to MHD}

Initially the entropic LB scheme for Navier-Stokes flows worked with the full distribution function $f_{i}$ [9-13]. This led to a discrete $\mathrm{H}$-theorem and the need to determine at each lattice site and for every time step the solution of a Newton-Raphson iterative procedure so that one remains on a constant entropy surface. This is computationally expensive $[12,13]$ for $3 \mathrm{D}$ turbulent flows. Recently the Karlin group 6, 7] separated the scalar lattice Boltzmann distribution into various moment-related groups and sort an algebraically based entropic parameter for the moment groups 
above the conserved and shear/stress moments. In particular, they formed 3 subgroups for the full $f_{i}$

$$
f_{i}=k_{i}+s_{i}+h_{i} \quad, \quad i=0 . .8 .
$$

Here the $k_{i}$ distributions correspond to the conserved moments, the $s_{i}$ distributions correspond to the stress/shear moments, and finally the $h_{i}$ distributions correspond to the remaining higher order moments.

For the $k_{i}$ distributions

$$
k_{i}=\sum_{j=0}^{8} \sum_{m=0}^{2} \mathrm{~T}_{i m}^{-1} \mathrm{~T}_{m j} f_{j} \quad, \quad i=0 . .8
$$

since there are 3 conserved moments, so the $m$-summation runs from $m=0,1,2$. Now the $s_{i}$ distributions corresponding to the stress/shear moments will come from the deviatoric stress, the trace of the stress tensor and 3rd order moments. We are at liberty to choose the building blocks of the $s_{i}$. Here we let the building blocks be the deviatoric stress and the trace of the stress tensor

$$
s_{i}=\sum_{j=0}^{8} \sum_{m=3}^{5} \mathrm{~T}_{i m}^{-1} \mathrm{~T}_{m j} f_{j} \quad, \quad i=0 . .8 .
$$

where the moments $\mathrm{m}=3,4,5$ are each 2 nd order moments in the D2Q9 model. Finally the remaining moments $\mathrm{m}=6,7,8$ are covered by the $h_{i}$

$$
h_{i}=\sum_{j=0}^{8} \sum_{m=6}^{8} \mathrm{~T}_{i m}^{-1} \mathrm{~T}_{m j} f_{j} \quad, \quad i=0 . .8 .
$$

A tunable parameter $\gamma$ is introduced to replace the relaxation rates for the higher order subgroup $h_{i}$, relaxation rates that do not affect the transport coefficients under Chapman-Enskog expansions 27]. In particular, instead of the standard LB post-collision distributions

$$
f_{i}^{\prime} \equiv f_{i}(t+1)=f_{i}+2 \beta\left(f_{i}^{(\mathrm{eq})}-f_{i}\right)
$$

$$
\text { we consider } \quad f_{i}^{\prime}=f_{i}-2 \beta \Delta s_{i}-\beta \gamma \Delta h_{i} \text {. }
$$

$\beta$ is related to the kinematic viscosity : $\nu=\frac{1}{6}\left(\frac{1}{\beta}-1\right)$ and we let $\Delta s_{i}=s_{i}-s_{i}^{(\mathrm{eq})}$ and $\Delta h_{i}=$ $h_{i}-h_{i}^{(\mathrm{eq})}$. For the conserved moments, of course, $\Delta k_{i}=k_{i}-k_{i}^{(\mathrm{eq})}=0$.

We wish to maximize the entropy $S[f]$

$$
S[f]=-\sum_{i} f_{i} \ln \left(\frac{f_{i}}{w_{i}}\right) .
$$

To do this, the entropy is written in terms of the post-collisional state and the $\gamma$ parameter. The critical point of the entropy [6,7] determines the tunable parameter $\gamma$ from

$$
\sum_{i} \Delta h_{i} \log \left(1+\frac{(1-\beta \gamma) \Delta h_{i}-(2 \beta-1) \Delta s_{i}}{f_{i}^{(\mathrm{eq})}}\right)=0
$$


Unfortunately, it is a rather computationally expensive root-finding procedure to determine $\gamma(\vec{x}, t)$ at every lattice grid point at every LB time step. However, Karlin et. al 6, 7] noted that if one invokes the simple small argument expansion $\log (1+x)=x+\ldots$ then the entropic factor can be determined algebraically. Since this is an approximation, we shall denote this entropic factor by $\gamma^{*}$, with

$$
\begin{gathered}
\qquad \gamma^{*}=\frac{1}{\beta}-\left(2-\frac{1}{\beta}\right) \frac{\langle\Delta s \mid \Delta h\rangle}{\langle\Delta h \mid \Delta h\rangle} \\
\text { where the inner product }\langle A \mid B\rangle=\sum_{i} \frac{A_{i} B_{i}}{f_{i}^{(\mathrm{eq})}} .
\end{gathered}
$$

Using this $\gamma^{*}$ in the new post-collisional state (Eq. 16) one has achieved a maximal entropy state. The Karlin group have successfully benchmarked this approximation for the tunable parameter $\gamma^{*}(\vec{x}, t)$ in various 2D and 3D Navier-Stokes simulations 6, 7]. One way to view this entropic algorithm is to consider it a dynamical subset of MRT - dynamical since the entropic parameter is tuned at every lattice point and every time step algebraically as opposed to the static relaxation times of a typical MRT simulation.

Clearly, this analysis can not simply carry over to LB-MHD with possible non-positive vector magnetic distributions for magnetic field reversals. Hence we make the ansatz for our partial entropic algorithm that the entropic parameter in LB-MHD will still be determined by Eq. (19) for the corresponding LB-MHD $\Delta h$ and $\Delta s$. The validity of our ansatz will now be tested against the Orszag-Tang vortex simulations [1].

Thus our partial entropic LB-MHD algorithm consists of the following steps (c.f., Karlin et. al. 6]:

1. Compute the conserved moments $(\rho, \mathbf{u}, \mathbf{B})($ Eq. 6, 9,10$)$

2. Evaluate the equilibria $\left(f_{i}^{(\mathrm{eq})}(\rho, \mathbf{u}, \mathbf{B}), \vec{g}_{k}^{(\mathrm{eq})}(\rho, \mathbf{u}, \mathbf{B})\right)$ (Eq. 4 )

3. Compute $s$ and $s^{(\mathrm{eq})}(\mathrm{Eq} \cdot 12$ ??)

4. Compute $\Delta s_{i}=s_{i}-s_{i}^{(\mathrm{eq})}$

5. Compute $\Delta h_{i}=h_{i}-h_{i}^{(\mathrm{eq})}=f_{i}-f_{i}^{(\mathrm{eq})}-\Delta s_{i}$

6. Evaluate $\gamma^{*}$ (Eq. 19)

7. Relax (Collide): $f_{i}^{\prime}$ (Eq. 16, and corresponding $\vec{g}_{k}^{\prime}$.

Standard LB-MHD is recovered when the entropy parameter is constant and equal to 2 : $\gamma^{*}(\vec{x}, t)=$ const.$=2$. However the effect of working with the maximal entropy state for the particle distribution function $f_{i}$ will have direct effects on the evolution of the magnetic field distribution $\vec{g}_{i}$ due to the coupling of the $\vec{B}$-field in the relaxation distribution function $f^{(\text {eq) }}$ as well as the coupling of the fluid velocity $\vec{u}$ in $\vec{g}^{\text {(eq) }}$.

\section{Partially Entropic LB-MHD Simulation of the Orszag- Tang Vortex [1]}

Since standard MRT-LB-MHD is recovered when the entropic parameter $\gamma^{*}(\vec{x}, t)=2=$ const. one can readily see the effect of partial entropic stabilization in the variation of $\gamma^{*}$ from 2.0. We consider the Orszag-Tang vortex [1] and qualitatively compare our entropic LB simulations with Ref. [1]. 

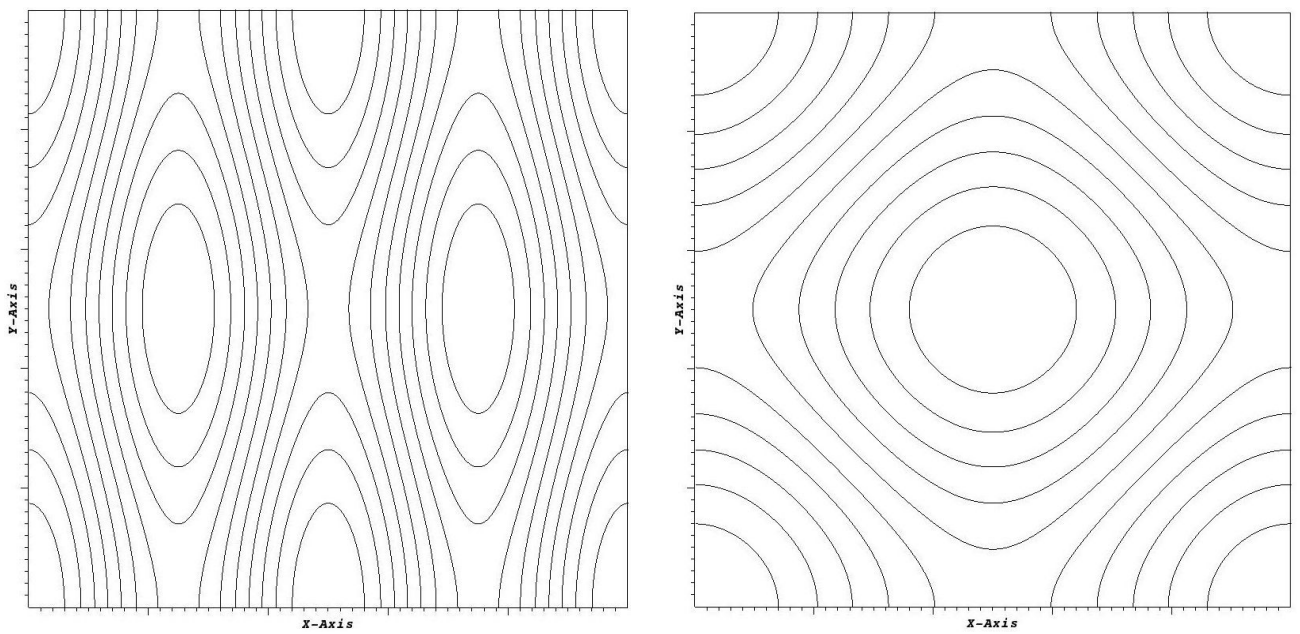

Figure 2: Initial profile of the (a) current and (b) vorticity contours respectively from the partial entropic LB-MHD code.

Here we show the physics recovered by the variations in the partially entropic parameter $\gamma^{*}$ and its variations away from the MRT value of $\gamma^{*}(\vec{x}, t) \equiv 2.0$ for the Orszag-Tang vortex. We qualitatively compare our simulations with the Orszag-Tang [1] profile. Consider the following initial profiles, Fig. 2 .

$$
\begin{gathered}
\vec{u}(x, y, t=0)=U_{0}[\sin (y) \hat{x}-\sin (x) \hat{y}] \\
\vec{B}(x, y, t=0)=B_{0}[\sin (y) \hat{x}-\sin (2 x) \hat{y}]
\end{gathered}
$$

with $U_{0}=B_{0}=6.1 \times 10^{-3}$ and $\nu=\eta=0.005$. These correspond to the profiles in 1 with viscosity $\nu$, resistivity $\eta$ and Reynold's number 1250. Snapshots of the current are shown in Fig. 3 - these are to be compared with Fig.7 of 11. Similarly, snapshots of the vorticity are shown in Fig. 4- and should be compared to those of Fig 8 in 1 . One finds excellent qualitative agreement with [1].

In Fig. 5 we plot the corresponding $2 \mathrm{D}$ entropy parameter $\gamma^{*}(x, y)$ at these two time snapshot, $t=26 \mathrm{~K}$ and $t=52 \mathrm{~K}$ iterations. The lattice points at which $\gamma^{*}(x, y) \neq 2$ correspond to spatial positions in which there are effects of our partial entropic LB-MHD algorithm. The energy over time for the Orszag-Tang vortex at $\nu=\eta=0.02$ is shown in fig. 6 for comparison against Fig. 5 in [1].

\section{Conclusion}

We have extended the Karlin 6, 7. entropic Navier-Stokes algorithm to LB-MHD and tested the ensuing model on an Orszag-Tang vortex. We considered the D2Q9 model for both the particle and vector magnetic field distributions. The partial entropy algorithm is applied only to the particle distributions while in using a vector distribution for the magnetic field we are automatically en-

forcing the $\boldsymbol{\nabla} \cdot \vec{B}=0$ constraint while permitting magnetic field reversals. The algorithm extends immediately to $3 \mathrm{D}$, but because of the much greater computational costs we have restricted our 

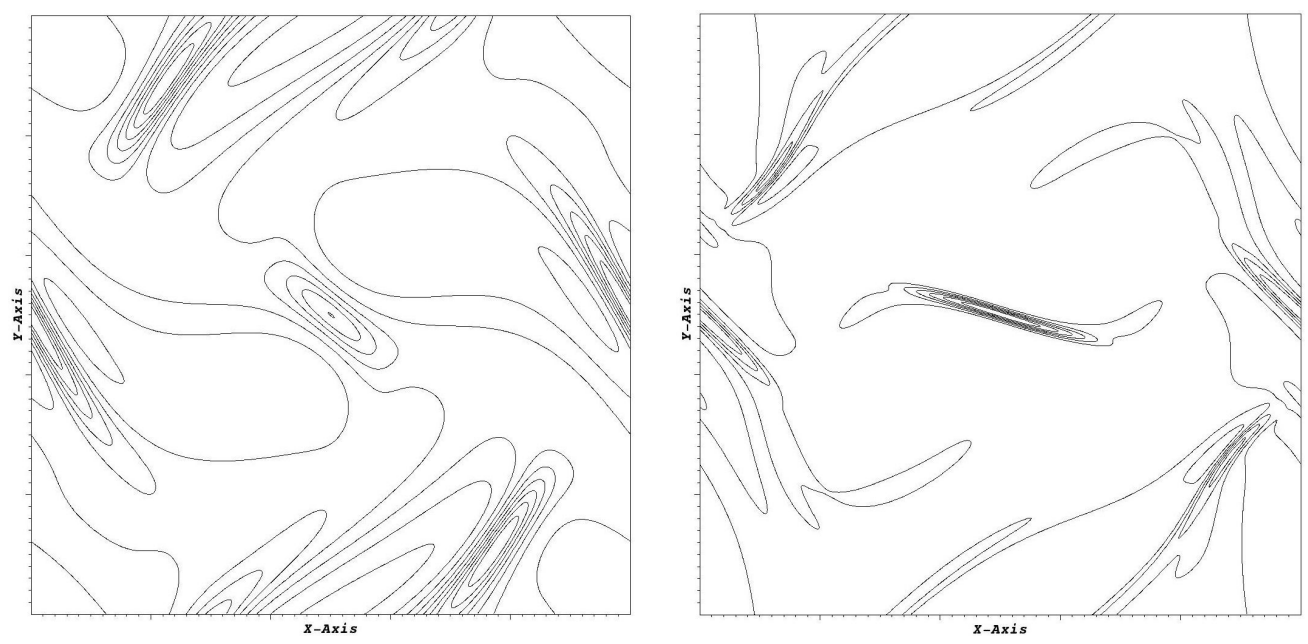

Figure 3: Snapshot of the current contours from the partial entropic LB-MHD code on a grid of $1024^{2}$ with $\nu=\eta=0.005$ (a) at time $=26 \mathrm{k}$, (b) at time $=52 \mathrm{k}$ (to be compared with Orszag-Tang [1], Fig. 7)
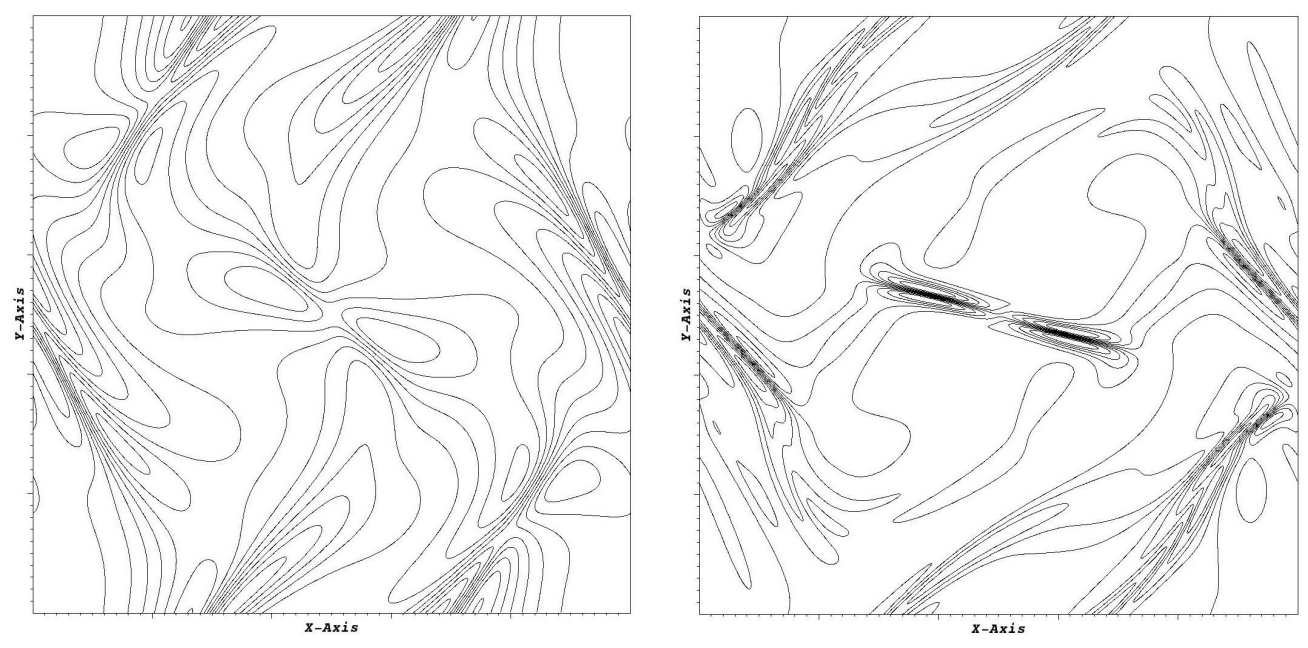

Figure 4: Snapshot of the vorticity contours from the partial entropic LB-MHD code on a grid of $1024^{2}$ with $\nu=\eta=0.005$ (a) at time $=26 \mathrm{k}$, (b) at time $=52 \mathrm{k}$ (to be compared with Orszag-Tang [1], Fig. 8) 

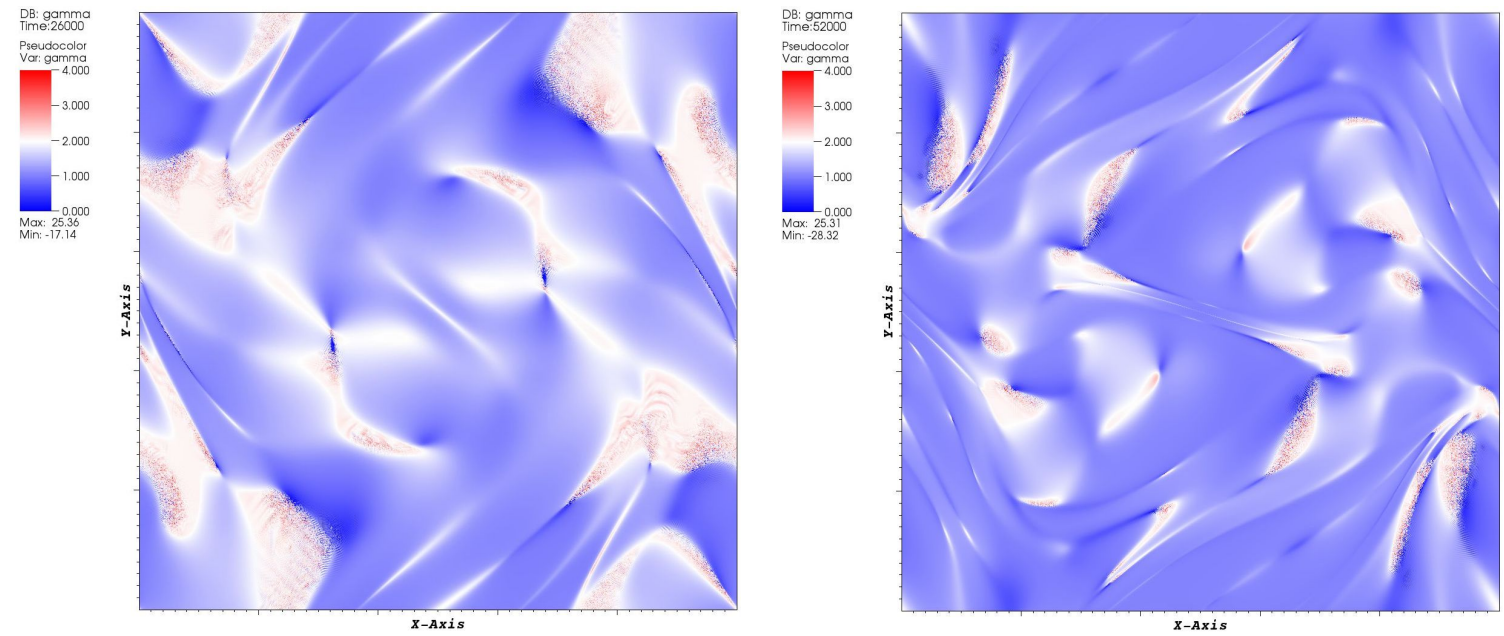

Figure 5: Plot of the entropic parameter $\gamma^{*}(x, y)$ after (a) 26k timesteps and (b) 52k timesteps on a $1024^{2}$ grid with $\nu=\eta=0.005$. $\gamma^{*}=2.0$ corresponds to ordinary LB-MHD. Lattice points with $\gamma^{*} \neq 2.0$ correspond to the stabilizing effects of the partial entropic LB-MHD algorithm.

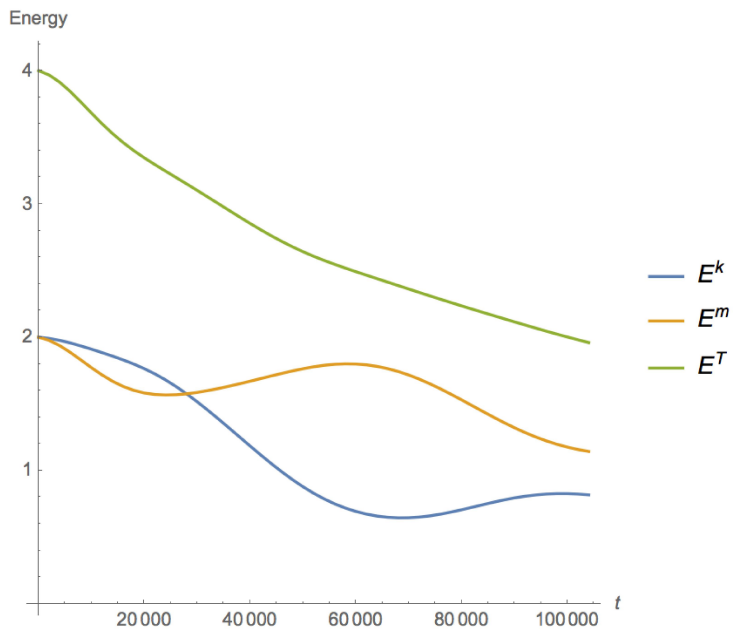

Figure 6: Plot of kinetic $\left(E^{k}\right)$, magnetic $\left(E^{m}\right)$, and total $\left(E^{T}\right)$ energies as a function of timesteps $t$ for the partial entropic LB-MHD code on a grid of $1024^{2} . \nu=\eta=0.02$ for comparison against Orszag and Tang's 11 Fig. 5. 
simulations to 2D. In 2D MHD one can still capture turbulence and the generation of small scale motions since in 2D MHD energy cascades to small scales. We have found good agreement with the CFD simulations of Orszag and Tang 1. Moreover the extreme parallelization of this partial entropic LB-MHD algorithm is retained since this algebraic entropic parameter $\gamma^{*}$ is determined purely from local information at each lattice site. The accuracy of the under-resolved Navier-Stokes simulations of Bösch et. al. 20] portend that this new (partial) entropy method could be a possible subgrid model in itself. This partial entropic LB-MHD algorithm is a subset of MRT models in which there is now a dynamical relaxation rate determined for quasi-stabilization of the fluid flow by a well-defined procedure as opposed to the standard static MRT relaxation rates.

\section{Acknowledgments}

This work was supported by an NSF grant 131424. The computations were performed on DoD Supercomputer Topaz.

\section{References}

[1] Steven A. Orszag and Cha-Mei Tang. Small-scale structure of two-dimensional magnetohydrodynamic turbulence. Journal of Fluid Mechanics, 90(1):129143, 1979.

[2] Sauro Succi. The lattice Boltzmann equation: for fluid dynamics and beyond. Oxford university press, 2001.

[3] Siarhei Khirevich, Irina Ginzburg, and Ulrich Tallarek. Coarse- and fine-grid numerical behavior of mrt/trt lattice-boltzmann schemes in regular and random sphere packings. Journal of Computational Physics, 281(Supplement C):708 - 742, 2015.

[4] Dominique d'Humires, Irina Ginzburg, Manfred Krafczyk, Pierre Lallemand, and Li-Shi Luo. Multiple-relaxation-time lattice boltzmann models in three dimensions. Philosophical Transactions: Mathematical, Physical and Engineering Sciences, 360(1792):437-451, 2002.

[5] Kannan N. Premnath and John Abraham. Three-dimensional multi-relaxation time (mrt) lattice-boltzmann models for multiphase flow. Journal of Computational Physics, 224(2):539 $-559,2007$.

[6] Fabian Bösch, Shyam S Chikatamarla, and Ilya V Karlin. Entropic multirelaxation lattice boltzmann models for turbulent flows. Physical Review E, 92(4):043309, 2015.

[7] Ilya V Karlin, Fabian Bösch, and Shyam S Chikatamarla. Gibbs' principle for the lattice-kinetic theory of fluid dynamics. Physical Review E, 90(3):031302, 2014.

[8] SS Chikatamarla and IV Karlin. Entropic lattice boltzmann method for turbulent flow simulations: Boundary conditions. Physica A: Statistical Mechanics and its Applications, 392(9):1925-1930, 2013.

[9] Bruce M Boghosian, Jeffrey Yepez, Peter V Coveney, and Alexander Wager. Entropic lattice boltzmann methods. Proceedings of the Royal Society of London A: Mathematical, Physical and Engineering Sciences, 457(2007):717-766, 2001. 
[10] Bruce M Boghosian, Peter J Love, Peter V Coveney, Iliya V Karlin, Sauro Succi, and Jeffrey Yepez. Galilean-invariant lattice-boltzmann models with h theorem. Physical Review E, 68(2):025103, 2003.

[11] Bruce M Boghosian, Peter J Love, Jeffrey Yepez, and Peter V Coveney. Galilean-invariant multi-speed entropic lattice boltzmann models. Physica D: Nonlinear Phenomena, 193(1):169181,2004 .

[12] Brian Keating, George Vahala, Jeffrey Yepez, Min Soe, and Linda Vahala. Entropic lattice boltzmann representations required to recover navier-stokes flows. Physical Review E, $75(3): 036712,2007$.

[13] G Vahala, B Keating, M Soe, J Yepez, L Vahala, and S Ziegeler. Entropic, les and boundary conditions in lattice boltzmann simulations of turbulence. The European Physical JournalSpecial Topics, 171(1):167-171, 2009.

[14] Christopher Flint, George Vahala, Linda Vahala, and Min Soe. A 9-bit multiple relaxation lattice boltzmann magnetohydrodynamic algorithm for $2 \mathrm{~d}$ turbulence. Computers $\&$ Mathematics with Applications, 72(2):394-403, 2016.

[15] Christopher Flint, George Vahala, Linda Vahala, and Min Soe. Magnetic field stabilization of a two-dimensional fluid jet: a multiple relaxation lattice boltzmann simulation. Radiation Effects and Defects in Solids, 170(5):429-438, 2015.

[16] Paul J Dellar. Bulk and shear viscosities in lattice boltzmann equations. Physical Review E, 64(3):031203, 2001.

[17] Paul J Dellar. Incompressible limits of lattice boltzmann equations using multiple relaxation times. Journal of Computational Physics, 190(2):351-370, 2003.

[18] Paul J Dellar. Moment equations for magnetohydrodynamics. Journal of Statistical Mechanics: Theory and Experiment, 2009(06):P06003, 2009.

[19] Kannan N Premnath, Martin J Pattison, and Sanjoy Banerjee. Dynamic subgrid scale modeling of turbulent flows using lattice-boltzmann method. Physica A: Statistical Mechanics and its Applications, 388(13):2640-2658, 2009.

[20] Fabian Bösch, Shyam S. Chikatamarla, and Ilya Karlin. Entropic multi-relaxation models for simulation of fluid turbulence. ESAIM: Proc., 52:1-24, 2015.

[21] MJ Pattison, KN Premnath, NB Morley, and MA Abdou. Progress in lattice boltzmann methods for magnetohydrodynamic flows relevant to fusion applications. Fusion Engineering and Design, 83(4):557-572, 2008.

[22] Benjamin M Riley, Jacques C Richard, and Sharath S Girimaji. Assessment of magnetohydrodynamic lattice boltzmann schemes in turbulence and rectangular jets. International Journal of Modern Physics C, 19(08):1211-1220, 2008.

[23] Paul J Dellar. Lattice kinetic schemes for magnetohydrodynamics. Journal of Computational Physics, 179(1):95-126, 2002. 
[24] Paul J. Dellar. Lattice boltzmann formulation for braginskii magnetohydrodynamics. Computers $\& 3$ Fluids, 46(1):201 - 205, 2011. 10th ICFD Conference Series on Numerical Methods for Fluid Dynamics (ICFD 2010).

[25] M'hamed Bouzidi, Dominique d'Humières, Pierre Lallemand, and Li-Shi Luo. Lattice boltzmann equation on a two-dimensional rectangular grid. Journal of Computational Physics, 172(2):704-717, 2001.

[26] Pierre Lallemand and Li-Shi Luo. Theory of the lattice boltzmann method: Dispersion, dissipation, isotropy, galilean invariance, and stability. Physical Review E, 61(6):6546, 2000.

[27] S.B. Pope. Turbulent Flows. Cambridge University Press, 2000. 